\title{
$I_{K s}$ Computational Modeling to Enforce the Investigation of D242N, a $K_{V} 7.1$ LQTS Mutation
}

\author{
Chiara Bartolucci ${ }^{*}$, Cristina Moreno ${ }^{2 *}$, Anna Oliveras ${ }^{3}$, Carmen Muñoz $^{4}$, Alicia de la Cruz ${ }^{2}$, Diego \\ A. Peraza ${ }^{2}$, Juan R Gimeno ${ }^{4}$, Mercedes Martín-Martínez ${ }^{5}$, Stefano Severi ${ }^{1}$, Antonio Felipe ${ }^{3}$, \\ Pier D Lambiase ${ }^{6}$, Teresa Gonzalez ${ }^{2}$, Carmen Valenzuela ${ }^{2}$ \\ ${ }^{1}$ University of Bologna, Bologna, Italy \\ ${ }^{2}$ Instituto de Investigaciones Biomédicas Alberto Sols CSIC-UAM, Madrid, Spain \\ ${ }^{3}$ Universitat de Barcelona, Barcellona, Spain \\ ${ }^{4}$ Hospital Universitario Virgen de la Arrixaca de Murcia, Spain \\ ${ }^{5}$ Instituto de Química Médica CSIC, Madrid, Spain \\ ${ }^{6}$ University College London, London, UK
}

\begin{abstract}
A KCNQ1 mutation, D242N, was found in a pair of twins and characterized at the cellular level. To investigate whether and how the mutation causes the clinically observed lost adaptation to fast heart rate, we performed a computational study.

Firstly, we identified a new $I_{K s}$ model based on voltage clamp experimental data. Then we included this formulation in the human action potential model of O'Hara Rudy (ORd) and simulated the effects of the mutation. We also included adrenergic stimulation to the action potential, since the basal adrenergic tone is likely to affect the influence of $I_{K s}$ on QTc in vivo. Finally, we simulated the pseudo-ECG, taking into account the heterogeneity of the cardiac wall.

At the basal rate (60bpm), the mutation had negligible effects for all cell types, whereas at the high rate (180bpm), with concomitant $\beta$-adrenergic stimulation (mimicking exercise conditions), the mutant AP failed to adapt its duration to the same extent as the wild-type AP (e.g. $281 \mathrm{~ms}$ vs. $267 \mathrm{~ms}$ in $M$ cells), due to a smaller amount of $I_{K s}$ current.

Pseudo-ECG results show only a slight rate adaptation, and the simulated QTc was significantly prolonged from $387 \mathrm{~ms}$ to $493 \mathrm{~ms}$, similar to experimental recordings.
\end{abstract}

\section{Introduction}

Long QT syndrome (LQTS) is one of the heart's primary electrical disorders, predisposing the affected individuals to sudden cardiac death caused by ventricular arrhythmias. The prolonged (usually $>480 \mathrm{~ms}$ ) corrected
QT interval (QTc) in these patients is caused by a delayed repolarization of the cardiac action potential (AP). Many genes are associated with LQTS: the KCNQ1 gene, which causes LQTS type 1 (LQTS1), accounts for more than $30 \%$ of the identified pathological genetic variants and mutations in LQTS. The KCNQ1 gene codes for $\mathrm{K}_{\mathrm{V}} 7.1$ channels which, after assembly with KCNE1 subunits, generate the slow delayed rectifier potassium current $\left(\mathrm{I}_{\mathrm{Ks}}\right)$ that greatly contributes to the repolarization of the cardiac AP [1].

$\mathrm{I}_{\mathrm{Ks}}$ becomes predominant at fast heart rates due to the slow deactivation kinetics, leading to an accumulation of $\mathrm{K}_{\mathrm{V}}$ 7.1-KCNE1 channels in the activated-closed state, facilitating channel opening when required [2]. The finding that exercise and emotional stress trigger arrhythmias in more than $80 \%$ of the LQTS1 patients demonstrates the key role of this current during elevated heart rates [3].

The KCNQ1 mutation D242N was found in two patients (twins) with LQTS [4]. Experimental data show that mutant channels generate a smaller current at physiological membrane potentials, supporting the currents' major role in the LQTS phenotype of the patients.

In this study, we identify an $\mathrm{I}_{\mathrm{Ks}}$ computational model which fits the experimental electrophysiological data and use it to investigate the effects of the biophysical and cellular phenotype of the mutation D242N on the AP and the pseudo-ECG using the O'Hara-Rudy (ORd) human cardiac AP model.

\section{Methods}

\subsection{Electrophysiology technique}


Two 20-year-old dizygotic twins (one female and one male) were evaluated after presenting with episodes of syncope and one nocturnal seizure pre-diagnosis. Both twins were genetically examined and found to carry the KCNQ1 mutation D242N (chr11:2572053G >A, c. $724 \mathrm{G}>\mathrm{A}$ ), localized in the exon 5 .

To examine the D242N effects in the physiologically relevant $\mathrm{K}_{\mathrm{v}} 7.1$ channel complex, the wild type (WT) $\mathrm{K}_{\mathrm{v}} 7.1$ and the mutant $\mathrm{D} 242 \mathrm{~N}$ subunits were cotransfected with KCNE1.

Membrane currents were measured from transiently transfected COS7 cells using the perforated-patch configuration of the patch-clamp technique with amphotericin and recorded using an Axopatch 200B amplifier (Axon Instruments) as described in Moreno et al.'s 2015 work [5]. Currents were recorded at room temperature $\left(21-23^{\circ} \mathrm{C}\right)$ at a stimulation frequency of 0.03 $\mathrm{Hz}$.

\subsection{Computational analysis}

To evaluate the electrophysiological consequences of the D242N mutation at the cardiac AP level, we used the ORd human ventricular model [6]. We fitted a new $\mathrm{I}_{\mathrm{Ks}}$ formulation, based on data obtained in WT and D242N channels for the steady state activation parameters $\left(\mathrm{V}_{\mathrm{h}}\right.$ and $\mathrm{s}$, see followed equations) and the deactivation time constant (see Figure 2). Since the ORd $\mathrm{I}_{\mathrm{Ks}}$ formulation includes a square value of the gating variable xs, its time constant was doubled with respect to the fitted experimental values. Finally, the activation time constant and maximal conductance were tuned to reproduce the voltage-clamp recordings of $\mathrm{K}_{\mathrm{V}} 7.1$ current in the presence of KCNE1, both in WT and mutant heterozygous conditions at room temperature (Figure 2), and to obtain, in WT, the same peak current during the AP as in the ORd original model.

The scaling factor $\mathrm{Q} 10=3$ [5] was used to scale the $\mathrm{I}_{\mathrm{Ks}}$ time constant from room temperature $\left(23^{\circ} \mathrm{C}\right)$ to physiological $\left(36^{\circ} \mathrm{C}\right)$. The $\beta$-adrenergic stimulation was simulated according to the computational study of Severi et al. [8] by increasing $\mathrm{I}_{K s}$ (scaled by a factor of 2.66), $\mathrm{I}_{C a L}$ (scaled by a factor of 1.5), and $\mathrm{I}_{u p}$ (scaled by a factor of 1.5 - - and by manipulating the time constant. In order to speed up deactivation and slow down activation ([7-8]), the constant was decreased for negative potentials and increased for positive potentials (see Figure 2). The $\mathrm{I}_{K s}$ formulation used in the AP simulations is:

$$
\begin{gathered}
\mathrm{I}_{\mathrm{Ks}}=\mathrm{G}_{\mathrm{Ks}} \cdot \mathrm{K}_{\mathrm{SCa}} \cdot \mathrm{x}_{\mathrm{s}}^{2} \cdot\left(\mathrm{V}-\mathrm{E}_{\mathrm{Ks}}\right) \\
\mathrm{G}_{\mathrm{Ks}}=\left\{\begin{array}{cc}
0.0083 & \text { endo and } M \text { cells } \\
0.012 & \text { epi cells }
\end{array}\right.
\end{gathered}
$$

$$
\begin{aligned}
& \mathrm{K}_{\mathrm{sCa}}=1+\frac{0.6}{1+\left(\frac{3.8 * 10^{-5}}{\left[\mathrm{Ca}^{2+}\right]_{i}}\right)^{1.4}} \\
& \mathrm{E}_{\mathrm{Ks}}=\frac{R T}{F} \cdot \ln \left(\frac{\left[\mathrm{K}^{+}\right]_{o}+P R_{N a, K} \cdot\left[\mathrm{Na}^{+}\right]_{o}}{\left[\mathrm{~K}^{+}\right]_{i}+P R_{N a, K} \cdot\left[N a^{+}\right]_{i}}\right) \\
& R=8314 \mathrm{~J} / \mathrm{kmol} / \mathrm{K}, \quad T=310^{\circ} \mathrm{K}, \quad F=96485 \mathrm{coul} / \mathrm{mol} \\
& {[K]_{o}=4 \mathrm{mM}, \quad[\mathrm{Na}]_{o}=140 \mathrm{mM}, \quad P R_{N a, K}=0.0367} \\
& \frac{d x_{s}}{d t}=\frac{x_{s \infty}-x_{s}}{\tau_{s}} \\
& x_{s œ W T}=\sqrt{\frac{1}{1+e^{\frac{-(V-32.9)}{16.6}}}} \quad \tau_{s W T}=150 * e^{-\left(\frac{V}{100}\right)^{2}} \\
& x_{S \infty \mathrm{WT} / \mathrm{D} 242 \mathrm{~N}}=\sqrt{\frac{1}{1+e^{\frac{-(V-40.8)}{20.7}}}} \\
& \tau_{s \mathrm{WT} / \mathrm{D} 242 \mathrm{~N}}=25+\left(21 * e^{-\left(\frac{V-12}{70}\right)^{2}}\right)^{1.6} \\
& \tau_{s_{-} \beta a d r}=\tau_{s} *(1-0.003 *(V-12))
\end{aligned}
$$

Single cell simulations. Model differential equations were implemented in Matlab (Mathworks Inc., Natick, MA, USA) and solved with a variable-order solver (ode15s). Pacing at the same experimental frequencies was maintained until a steady state AP was reached, and the AP duration (APD) was measured as the interval between the AP upstroke and the $90 \%$ repolarization level $\left(\mathrm{APD}_{90}\right)$.

Multicellular simulations. A one-dimensional fiber (1.64 cm length) composed of 25 endo-, $30 \mathrm{M}-, 50 \mathrm{mid}-$ and 60 epi-cardial cells was simulated [9]. Midmyocardial cells were modeled with intermediate (between endo- and epi-) parameter values; the pseudoECG signal was computed upon pacing of the endocardial end of the tissue as previously described [10], using intracellular and extracellular conductivity of $2 \mathrm{~ms} / \mathrm{cm}$ and $8 \mathrm{~ms} / \mathrm{cm}$, respectively.

Model equations were translated into cellML language in the COR environment, and monodomain equations were solved with Chaste Software [11].

\section{Results}

\subsection{Electrophysiological results}

The patients' heterozygous conditions were mimicked 
by expressing $50 \%$ of the mutant channels.

Figure 1 (black) shows the experimental average of the WT and D242N traces obtained after applying the pulse protocol (shown at the top). The activation curves for mutant channels are mildly shifted toward positive potentials with respect to the WT channels. Additionally, the deactivation kinetics were faster for mutant channels than WT channels $(313.8 \pm 89.2 \mathrm{~ms}$ vs. $513.6 \pm 37.0 \mathrm{~ms}$, $\mathrm{n}=5 / 15, \mathrm{P}<0.05)($ Figure 2).
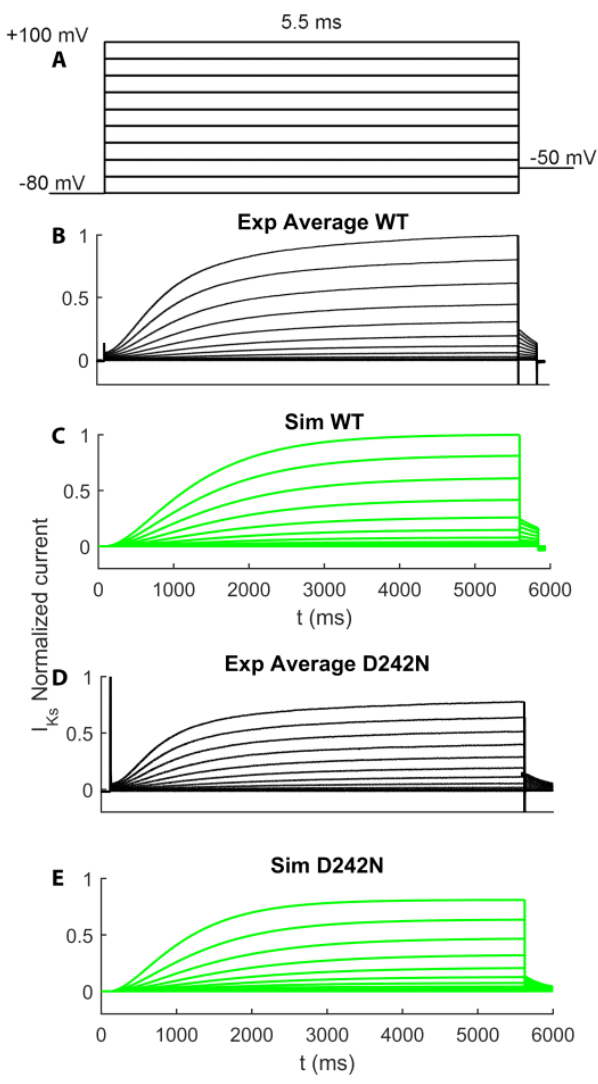

Figure 1. A: voltage clamp protocol; pulses were applied, in $10 \mathrm{mV}$-steps, from a holding potential of $-80 \mathrm{mV}$ to $+100 \mathrm{mV}$. Tail currents were recorded at $-50 \mathrm{mV}$ for mutant and $-40 \mathrm{mV}$ for WT. B and D (black): Average WT and D242N current records. C and E (green): Simulated WT and D242N currents.

\subsection{Computational results}

The results of the $\mathrm{I}_{\mathrm{Ks}}$ model identification based on the experimental data are shown in Figure 1, panels $\mathrm{C}$ and $\mathrm{E}$.

The ORd human ventricular cell model with the new $\mathrm{I}_{\mathrm{Ks}}$ formulation (see Methods) was used to examine the effects of the heterozygous condition on AP morphology in endocardial, epicardial, and $\mathrm{M}$ cells (only $\mathrm{M}$ cells are reported in Figure 3). At the basal rate $(60 \mathrm{bpm})$, the mutation had a negligible effect on all cell types. In contrast, at the higher rate (180bpm) with concomitant $\beta$ adrenergic stimulation (mimicking exercise conditions) the mutant AP failed to adapt its duration to the same extent as the WT AP (e.g. 281 vs. $267 \mathrm{~ms}$ in M cells, Figure 3, top panel) due to a smaller amount of $\mathrm{I}_{\mathrm{Ks}}$ current (Figure 3, middle panel). The impaired rate adaptation exhibited by the mutant was also evident when the rate dependence curve was computed $\left(\mathrm{APD}_{90}\right.$ vs. pacing cycle length, changing the $\%$ of $\beta$-adrenergic stimulation: no $\beta$ adr at $\mathrm{CL}=1000 \mathrm{~ms}, 25 \%$ at $\mathrm{CL}=700 \mathrm{~ms}, 50 \%$ at $\mathrm{CL}=500 \mathrm{~ms}, 75 \%$ at $\mathrm{CL}=400 \mathrm{~ms}$ and $100 \%$ at $\mathrm{CL}=333 \mathrm{~ms}$ ) (Figure 3, bottom panel).

At the cardiomyocyte level, the $\mathrm{I}_{\mathrm{Ks}}$ current amplitude increases with heart rate and $\beta$-adrenergic stimulation. Our simulation results suggest that both factors contribute equally to exercise adaptation since if rate is increased with no $\beta$-adrenergic stimulation, a small decrease in APD adaptation is observed (e.g. WT: from 356 to 298 $\mathrm{ms}$, D242N: from 359 to $305 \mathrm{~ms}$, at $180 \mathrm{bpm}$ ), and a similar effect is produced by simulation of $\beta$-adrenergic stimulation alone with no change in rate (WT: from 298 to $267 \mathrm{~ms}$, D242N: from 305 to $281 \mathrm{~ms}$ ).

In order to relate the modeled changes in $\mathrm{APD}_{90}$ to QTc, we computed pseudo-ECGs for heterozygous conditions. In Figure 4, the results of simulations run at exactly the same heart rates experimentally recorded in twin \#1 at baseline and under exercise (84bpm vs. $163 \mathrm{bpm}$ plus $\beta$-adrenergic stimulation) are shown. Only a slight rate adaptation is observed, and the simulated QTc was significantly prolonged from $387 \mathrm{~ms}$ to $493 \mathrm{~ms}$, similarly to experimental recordings.

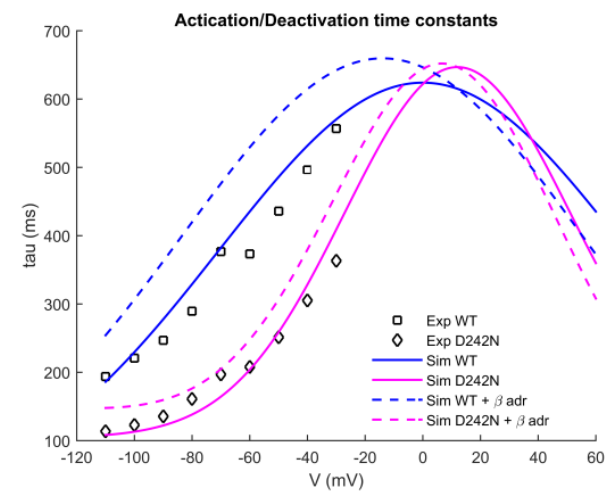

Figure 2. Experimental values and model fitting for the activation/deactivation time constant.

\section{Discussion and Conclusion}

The electrophysiological results suggest that, in cardiomyocytes, this mutation induces a delay in the repolarization phase of the cardiac AP. The delay ultimately triggers the prolongation of the QT interval, leading to the almost complete loss of adaptation to high heart rates which is detected in D242N-LQTS1 patients. 
The computational analysis confirmed that these mutant channels cannot respond properly to heart rate changes. It also provided a plausible explanation for the clinical manifestations of the mutation (only a slight decrease in the QT interval during exercise, with a consequent great increase in the QTc). While the mutation has negligible effects on the AP at baseline, a failure to adapt to exercise conditions was identified.
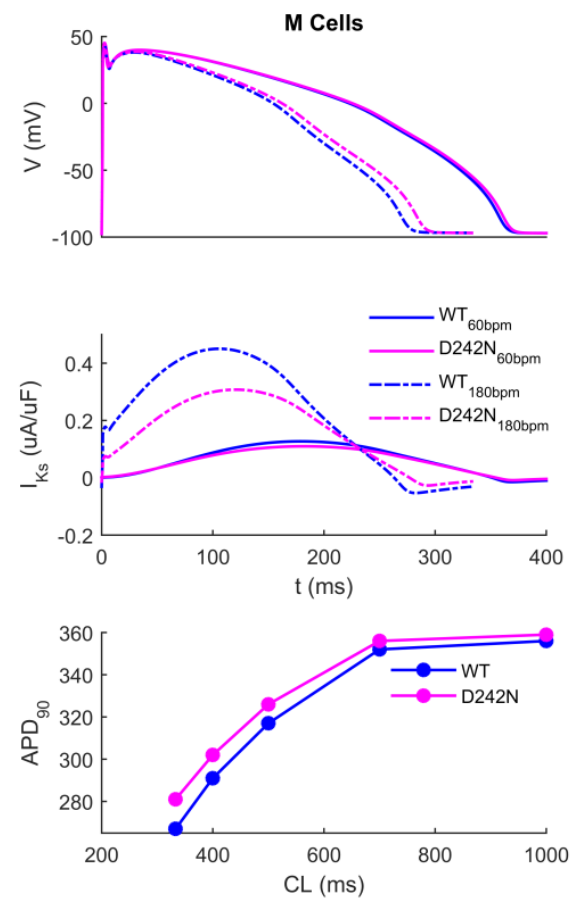

Figure 3. Action potentials (top), $\mathrm{I}_{\mathrm{Ks}}$ current (middle), and APD rate dependence (bottom) in WT and D242N conditions for ventricular M cells. Simulations were performed mimicking basal (60bpm) and exercise (180bpm and $\beta$-adrenergic stimulation) conditions.

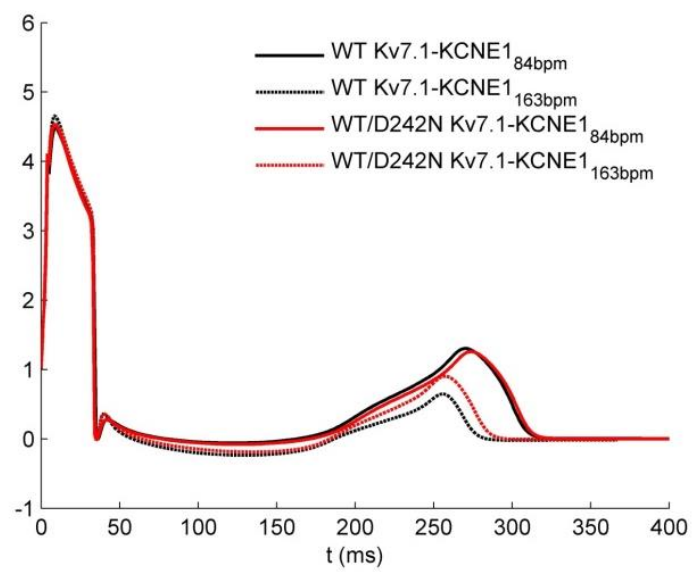

Figure 4. Pseudo-ECGs obtained in the $\mathrm{WT} \mathrm{K}_{\mathrm{V}}$ 7.1KCNE1 and in the WT/D242N K $7.1-\mathrm{KCNE} 1$ conditions in simulations run at exactly the same heart rates experimentally recorded in twin \#1 at baseline and under exercise $(84 \mathrm{bpm}$ vs. $163 \mathrm{bpm}$ plus $\beta$-adrenergic stimulation).

\section{References}

[1] Barhanin J, Lesage F, Guillemare E, Fink M, et al. $\mathrm{K}(\mathrm{V}) \mathrm{LQT} 1$ and $1 \mathrm{sK}(\mathrm{minK})$ proteins associate to form the $\mathrm{I}(\mathrm{Ks})$ cardiac potassium current. Nature 1996;384:78-80.

[2] Volders PG, Stengl M, van Opstal JM, Gerlach U, et al. Probing the contribution of IKs to canine ventricular repolarization: key role for betaadrenergic receptor stimulation. Circulation 2003;107:2753-60.

[3] Schwartz PJ, Priori SG, Spazzolini C, Moss AJ, et al. Genotype-phenotype correlation in the long-QT syndrome: gene-specific triggers for life-threatening arrhythmias. Circulation 2001;103:89-95.

[4] Moreno C, Oliveras A, Bartolucci C, Muñoz, C, et al. D242N, a Kv7.1 LQTS mutation uncovers a key residue for IKs voltage dependenc. J Mol Cell Cardiol 2017;110:61-69.

[5] Moreno C, de la Cruz A, Oliveras A, Kharche SR, et al. Marine n-3 PUFAs modulate IKs gating, channel expression, and location in membrane microdomains. Cardiovasc Res 2015;105:223-32

[6] O'Hara T, Virag L, Varro A, Rudy Y. Simulation of the undiseased human cardiac ventricular action potential: model formulation and experimental validation. PLoS Comput Biol 2011;7:e1002061.

[7] Severi S, Corsi C, Rocchetti M, Zaza A. Mechanisms of beta-adrenergic modulation of $\mathrm{I}(\mathrm{Ks})$ in the guinea-pig ventricle: insights from experimental and model-based analysis. Biophys $J$ 2009;96:3862-3872.

[8] Walsh KB, Begenisich TB, Kass RS. Beta-adrenergic modulation of cardiac ion channels. Differential temperature sensitivity of potassium and calcium currents. J Gen Physiol. 1989;93(5):841-54

[9] Glukhov AV, Fedorov VV, Lou Q, Ravikumar VK, et al. Transmural dispersion of repolarization in failing and nonfailing human ventricle. Circulation research 2010;106:981-991.

[10] Gima K, Rudy Y. Ionic current basis of electrocardiographic waveforms: a model study. Circ Res 2002;90:889-96

[11] Mirams GR, Arthurs CJ, Bernabeu MO, Bordas R, et al. Chaste: an open source C++ library for computational physiology and biology. PLoS Computational Biology 2013;9:e1002970.

Address for correspondence.

Stefano Severi stefano.severi@unibo.it

DEI, University of Bologna, Cesena,

Via Venezia 52, 47521, Cesena (FC), Italy 